\title{
Standard Model Picture with 61 Elementary Particles
}

\author{
Hermann Josef Scheuber \\ Department of Chemistry, ETHZ, Zurich, Switzerland \\ Email address: \\ hermann.scheuber@alumni.ethz.ch
}

To cite this article:

Hermann Josef Scheuber. Standard Model Picture with 61 Elementary Particles. International Journal of Applied Mathematics and Theoretical Physics. Vol. 4, No. 2, 2018, pp. 42-49. doi: 10.11648/j.ijamtp.20180402.12

Received: September 15, 2017; Accepted: January 28, 2018; Published: August 9, 2018

\begin{abstract}
In 1960 Gell-Mann completed the «Particle Zoo» with a picture simulating a 3-dimensional model: Spin, Strangeness and an oblique incident charge with 3 investigated Quarks constructed in 10 Baryons. Until now the CERN scientists have found 61 elementary particles, but only 17 Fundamental particles are listed in a brick box as «Standard model». But if the Quarks really exist, why not use Gell-Mann's picture which I tried to enhance with modern media. This explains why - in contrast to common practice - only pictures can be seen in this publication and NO Lagrange formula.
\end{abstract}

Keywords: Standard Model, QCD, 61 Elementary Particles, Particle Physics with Pictures

\section{Introduction}

If you have heard all about the fundamental particles in the standard model but have never seen how these 61 very small points could be integrated in a standard system matching all together, then you've come to the right place here to follow Gell-Mann, the investigator of the Quarks even sixty years ago - but since then no other picture exists. Only in the CERN 17 Fundamental particles have been found, building a brick box: the three upper quarks: up, charm and top; the three lower quarks: down, strange and bottom; the three neutrinos: Electron-neutrino, $\mathrm{Mu}-$ neutrino and Tau-neutrino; the three electrons: Electron, and again the couple $\mathrm{Mu}$ - and Tau-electron; until now also 12 half-spin particles, which results with the five 1 spin Bosons: (W+ and W- ) charge Ray, (8)Gluon(s), weak Z force, Photon \& Higgs in exactly 17 Fundamental particles. But as the particles with half-spin should be doubled by spin inversion (Pauli) and the quarks even tripled to get three distinguishable Quarks for the Baryons, the enlarged Standard Model will get 61 Elemental particles which until now are only known as a Lagrange formula with about 20 parameters. But as not everybody can imagine this formula, I tried to devise a picture step by step by using the free downloadable PC program
GeoGebra 5 on Windows 10. All pictures shown here are ones I constructed myself with the freely accessible data for spin \& charge and the strangeness to get a revolving Stereo picture. But as the publicizing possibilities are limited, the pictures here are restricted to momentarily chosen stationary ones. But nevertheless, even if it looks very complicated with the 7 circle planes, to expound off a Lagrange formula takes even longer [1-5] First, the CERN brick box of the 17 Fundamental particles had to be enhanced: the half spin fermions corresponding to Pauli by Spin inverting and QCD namely 3 distinguishable Quarks i.e. analogue to 3 imaginary colors making up white: green Quark - inversion - red Quark - QCD therefore blue Quark. The same also with the Antiquark colors: magenta - cyan blue - yellow. The Electron Neutrino gives the Antineutrino and the Electron with left spin - inversion - the right spin electron and at (Higgs)center inversion the positron. From the 8 Gluons there are 6 sitting on the horizontal Boson spin 1 and 2 ones on the vertical spin 1 i.e. on $\mathrm{W}-1$ and $\mathrm{W}+1$ respectively. The 61 particles can therefore be gotten from the following Figure 1. 


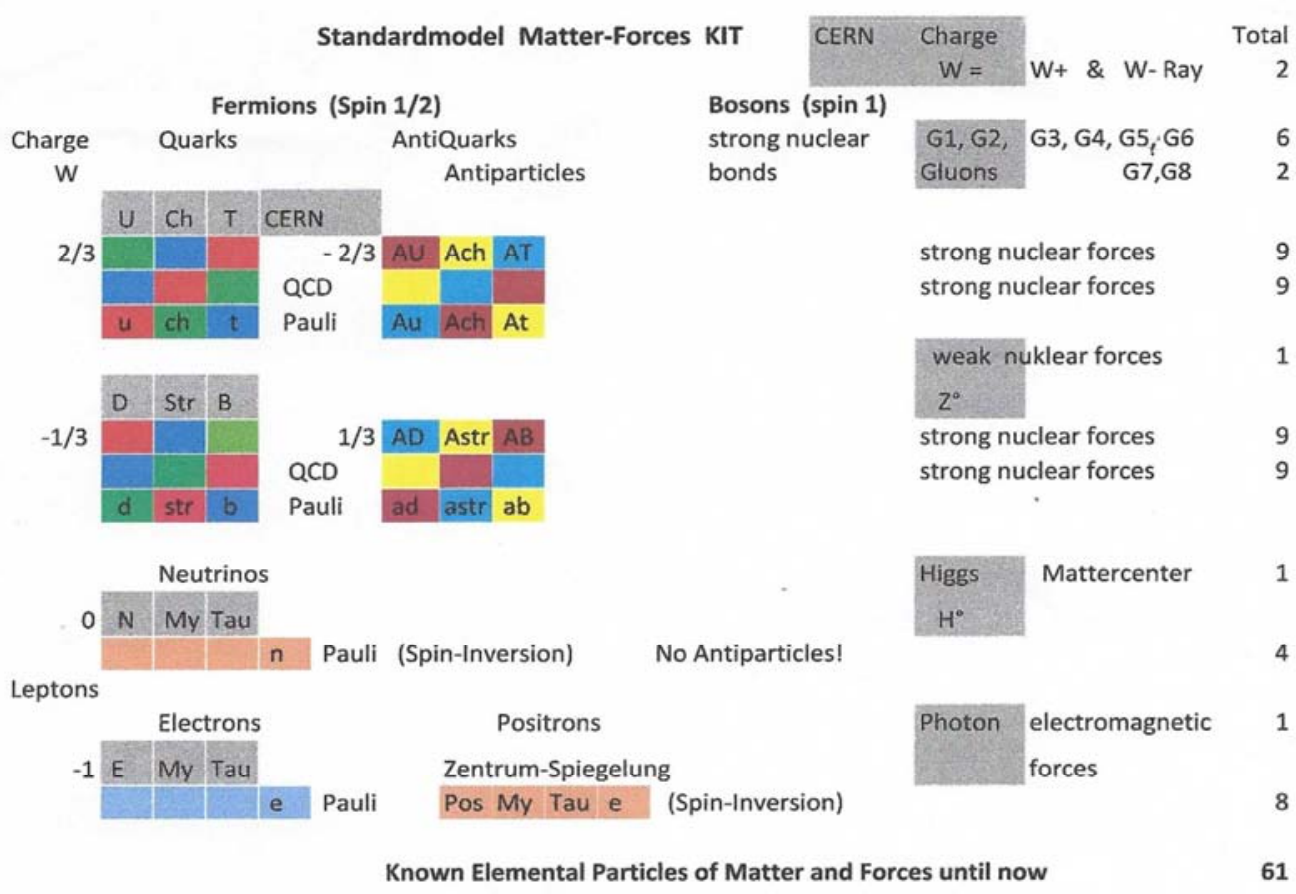

Figure 1. Completed Standard Model.

\section{Construction of the Models with Inverted Axes and Views}

\subsection{Input of Data with the GeoGebra Program 5}

The Basis, cleverly devised by Gell-Mann's eightfold way is the stereo coordinate system (spin-strangeness-charge) with the Neutron-Baryon and the modern Higgs-center Point (zero, zero, zero). So, we begin with the Spin and go to the next step: the half spin: left with the negative, left rotating i.e. the Mu-neutrino and right with the positive and bigger Tauneutrino; both sides united in a closed circle - thanks to GellMann, choosing the strangeness as planar dimension and the incident oblique charge to draw a similar stereo picture. But he constructed the picture without the later detected left rotating Neutrinos. As the Tau-neutrino should be right rotating (according to the symmetric picture system) there lies again more work for the investigation of the missing grey matter. So, Gell-Mann began first with the Mesons, the 2 Kaons with Strangeness +1 , the Zero Spinline on Strangeness 0 with the Pions Pionminus \& Pionplus, the Baryons with increasing charge: baryon -1 , Neutron 0 , Proton +1 and the baryon +2 , and today last but not least the Higgsparticle as Zero Stereo Coordination point. On the Strangeness -1 there are the other 2 Kaons and the 2 Sigmabaryons. So, we can connect the 4 Kaons and the 2 Pions to get a Meson Sextet between the strangeness axes $+1 \&-1$ [8]. We connect also, the Neutron line from the left to the right in the Neutrino half-spin circle. We get the cross points Antineutrino and the Electron Neutrino and enlarge the Sextet by the double + loaded Baryon double Delta on the strangeness axis 0 to get the oblique incident charge $+2,+1$ (= Positron), zero (=
Chargecenter). The Electron we can get from the Positron +1 by drawing the connection line through the Higgscenter. The Detail Figure 11 shows the exact construction with the same methods as Gell-Mann for the Baryon Decuplet. The Zero Chargecenter lies on Strangeness -1 and the Higgscenter on Strangeness 0. We fix the Electron, the Positron and draw the strangeness lines, connected by the half-spin right and left, and connect the other corners until we get exactly GellMann's Basic construction with the Omega Baryon on Strangeness -3 , containing 6 triangles with the 6 centers of gravity which we call the Quarks, namely Up and Down, Strange, and later the subsequently detected Charm, Bottom and Top. The reason for the name Strange lies therein that the + strangeness has changed with the - strangeness of the Charm; therefore, we take actual the spin-inversed particles to draw the half-spin circle of the same charge. The 3 Double (one $+\&$ the other - ) triangles in green, blue and red represent the 3 mass increasing Quark families. They have been projected onto the neutral plane to show the upper $(+)$ and lower (-) charge parts better. On this neutral plane there are also the weak power Bosons Photon and $\mathrm{Z}$ which we connect by the Boson 1 spin circle. One charge quant below the Neutrino neutral plane there lies the Muon and the Tauon or Tau - so we had better turn the axes and get a bit of strangeness for a real stereo picture. The figure you see is that the $\mathrm{W}+$ and the $\mathrm{W}$ - ray are connected to a charge axis with the two Quark half-spin levels on $+2 / 3$ and $-1 / 3$ charge level, so that we can draw the Electron half-spin circle on charge -1 because we have three -1 charged points: Electron, Muon and Tau. As the Standard Model proposes that each half spin point has its Antipoint, we get the Anti-Muon \& the Anti-Tau by inverting the signs or constructively by placing 
reflection rays through the Higgs-zero Point. Thus, we get the Anti-Electron as Positron half-spin circle and as fourth point on both circles the spin inverted Pauli counter electron and Anti-electron respectively. Therefore, there are 4 particles connected in both half-spin circles $\mathrm{W}+$ and $\mathrm{W}-$-: the couple Muon and Tau as well as the big Initialized Electron and the spin inverted, small initialized electron - like the Neutrino half-spin circle. As each half-spin point has its Antipoint, after marking the four center points with a white ball, we get the minus 2/3 half-spin circle with Anticharm, Antiup and Antitop and the other 1/3 Anti half-spin circle with Antistrange, Antidown and Antibottom. The center of gravity point of the green projected triangle on the neutral plane are also shown. According to Pauli, all these quarks must have their spin inverted counter Quarks, so that each levelled quark half-spin circle would get six different quarks, i.e. on the $-2 / 3$ Anti-spin-circle: additionally, Anti up, Anti charm and Anti top. But as Baryons can have three equal but distinguishable quarks there should be another three Quarks more in the spin circle, which can be done by "Quantum Chromatic Dynamic", abbreviated QCD. Therefore, we construct through the white bowls on the $\mathrm{W}+$ and $\mathrm{W}$ - ray at $2 / 3$ and $1 / 3$ these colored QCD's, because they consist of the same three colored Quarks: blue, green and red; and the antiquarks as yellow, magenta and cyan blue respectively; all three together result again in white; necessary for each existing Baryon containing these three different colored Quarks or three Antiquarks. We completed therefore the $-2 / 3$ counter Antiquarks with the antiquark colors and indicate the $\mathrm{W}+2 / 3$ counter Quarks with small initialized terms charm, up and top with the Quark colors. We continue with the W-1/3 counter Quarks small initialized down, strange and bottom and finish with the $\mathrm{W}+1 / 3$ counter Antiquarks: Antidown, Antistrange and Antibottom with the Antiquark colors also called "anti-green"," anti-red", "anti-blue", i.e. magenta, cyan-blue and yellow respectively. To demonstrate the two matching Quark spin-circles we connect the corresponding Quarks and see: The white balls are the spin-circle centers of the $\mathrm{W}+2 / 3$ and $\mathrm{W}+1 / 3$ lying on the $\mathrm{W}+$ ray and the spin circle centers of the $\mathrm{W}-1 / 3$ and $\mathrm{W}-2 / 3$ lying on the $\mathrm{W}$ - ray. On each Quark spin level lie 9 Quarks or 9 Antiquarks. The Quarks in the Baryons are fixed by Gluons. To place the Gluons, we rotate the axis again and add the six double colored gluon Bosons (one Quark color side and the other with Antiquark color) on the 1 Spin-circle and the other two as 4 and 6 anti-coloreds as $\mathrm{W}+1$ and $\mathrm{W}-1$; filling out the 8 Gluon-interstices with the 9 Quarks, to keep them bonded very strongly together in the Baryonic nucleus. At the end of the 7 planes of the Standard Model we can test the precision of the picture by rotating the axes and checking the Higgs crossing lines. But finally, we can turn and rotate the whole System and look at each particle with all the possibilities of the GeoGebra program e.g. checking Electrons, Neutrinos, Quarks, Gluons, Bosons and the Algebra points given by the 3-dimensional construction, enlarging \& so on. [11, 12]

\subsection{Construction Pictures}

\subsubsection{The Picture with Perpendicular Charge Axis (W+, Higgs, $W$-) Has 7 Planes}

The Construction can by clicking according [11] step by step be observed as well as printed out with the exact construction program.

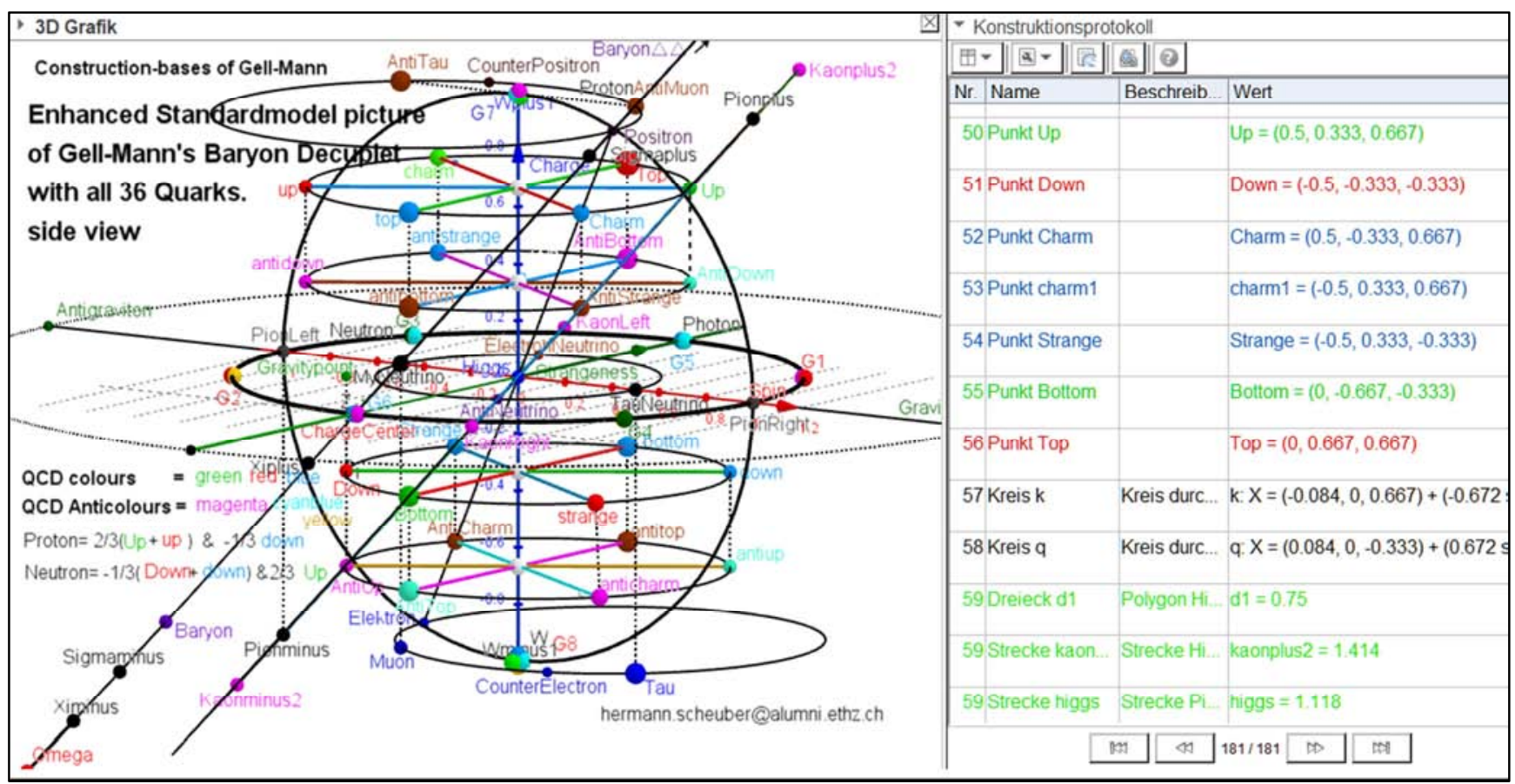

Figure 2. The Construction of the Standard Model with 61 Elementary QCD particles. 


\subsubsection{It Can Be Shown That the Quarks Are Really} Trigonal Points of Gravity

On Neutral plane, the 6 and 2 different Gluons on the horizontal \& vertical Boson spin 1 demonstrate the 3 families of the particle physics each one having 1 upper \& 1 lower Quarks.

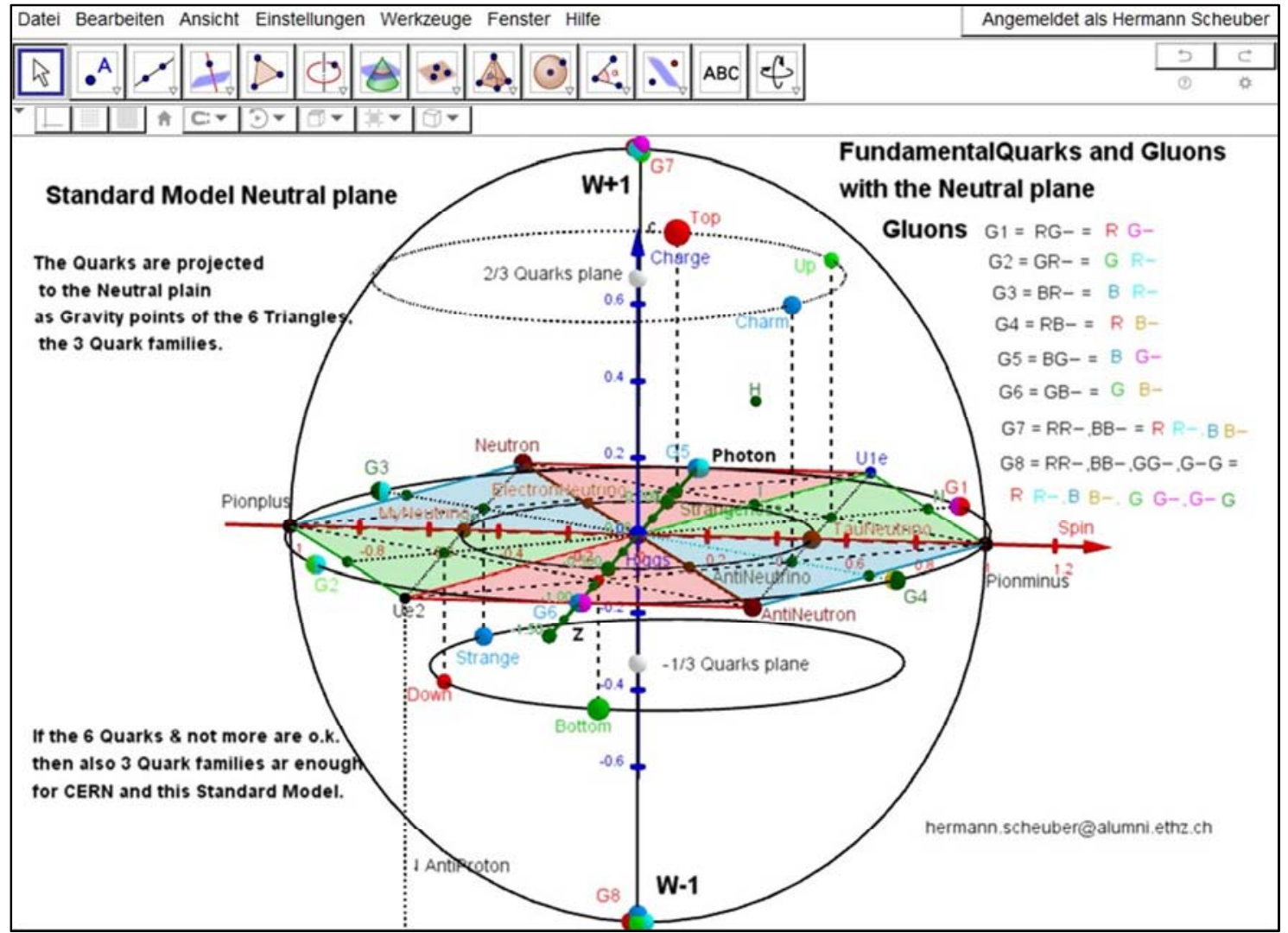

Figure 3. Shows the (projected) Neutral plane, the horizontal and the perpendicular 1 Spin Boson-circle for the 6 \& 2 Gluons.

2.3. Comparison of the Enhanced Vertical Model (Figure 6) with Gell-Mann's Meson Octet and Baryon Decuplet.

\subsubsection{Discussion of Gell-Mann's Quark Models (Figure 4} \& 5)

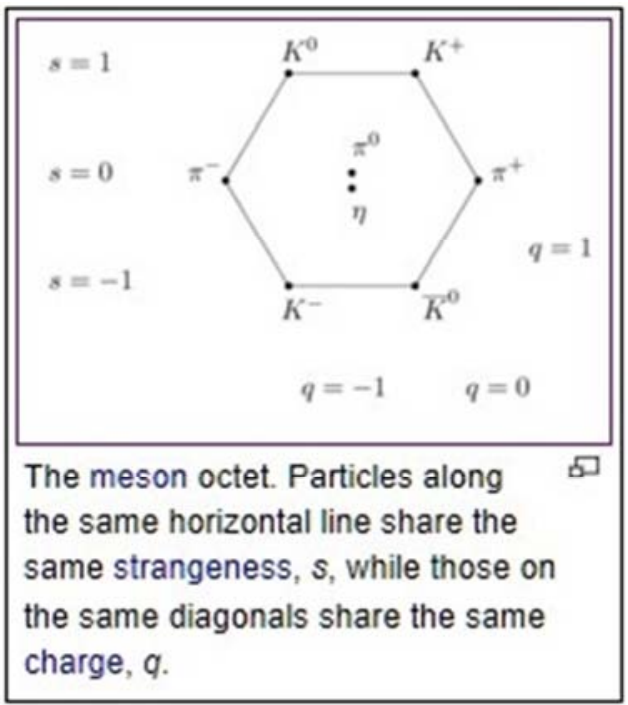

Figure 4. Gell-Mann's Meson octet.

Gell-Mann's meson octet shows that the 4 half-Spin Kaons have strangeness $(+\&-1)$ but both the 1 Spin Pions (Pion minus \& Pion plus) with strangeness 0 lay on the spin axis. Therefore, the charge Ray Q incidents between the Kaon K+ $\&$ the pion plus on the right side $(\mathrm{q}=+1=$ Positron $)$ to the left side between the Kaon $\mathrm{K}-\&$ the pion minus $(\mathrm{q}=-1=$ Electron). [6,9]

There are 2 Zero Points with different Strangeness.

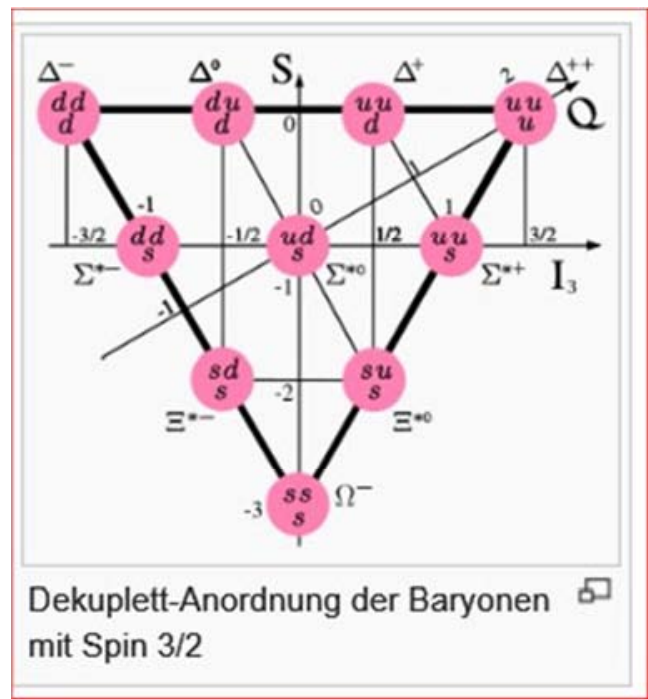

Figure 5. Gell-Mann's Baryon decuplet. 


\subsubsection{Showing the Same Construction of Figure 2, but} Horizontal (Without Visible Charge)

This picture (Figre 6) continues the Baryon decuplet as also the (6 and 2) Gluons investigated later in the same position of the axes (spin, Strangeness) without a visible stereo effect but with high symmetry.

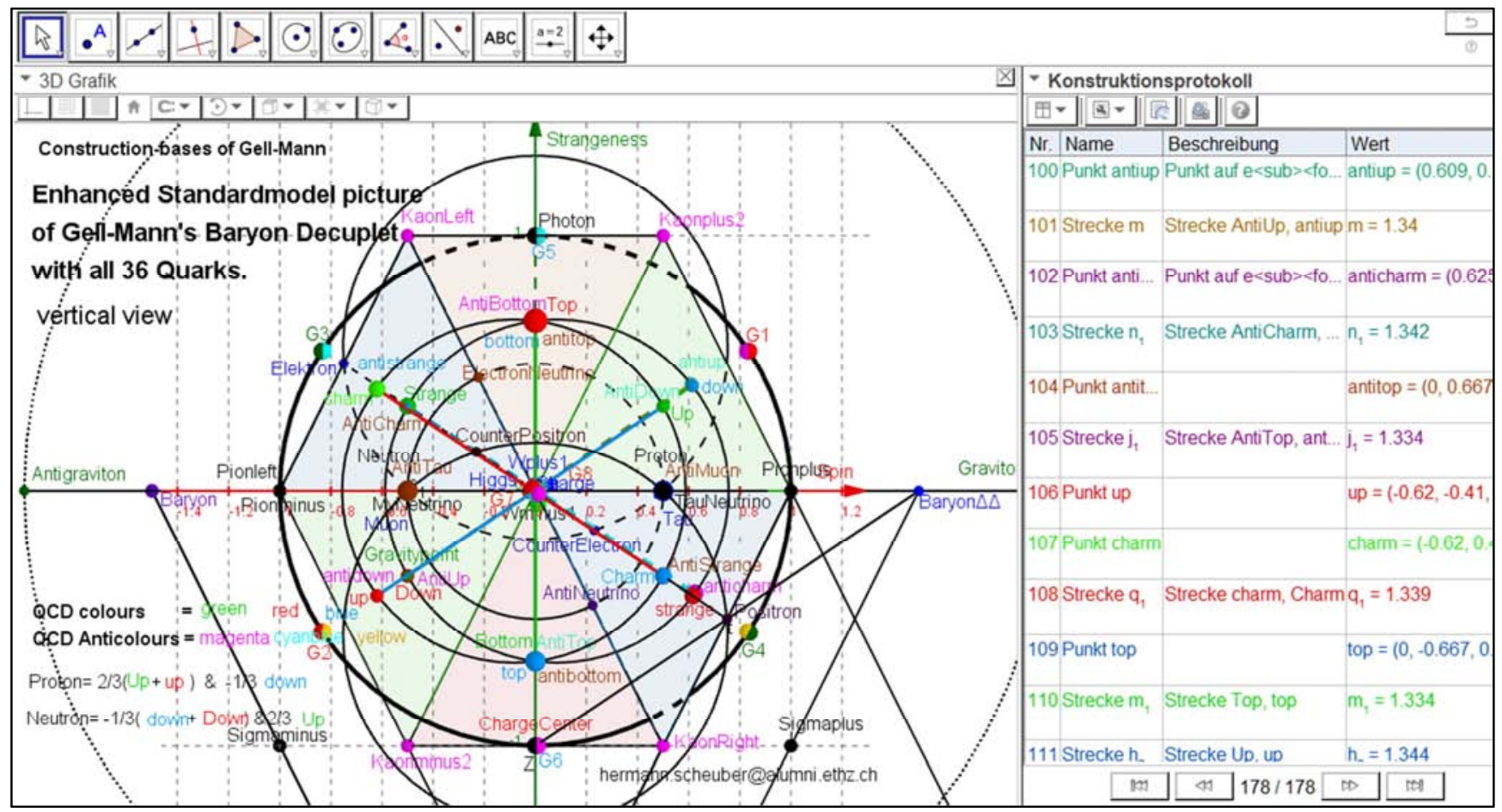

Figure 6. High Symmetry of the enhanced "Decuplet" with 61 Particles and Spin circles; the right side shows construction details (spin, strangeness, charge)

This picture shows that if the Quarks are real (and even the 6 and 2 Gluons) then it is also real that only three particle families can exist! So, a great problem of particle physics should be solved. (In the proved CERN Standard model there is no place for a forth family!)

\subsection{Model of the 61 Elementary Particles Without Disturbing Construction Details}

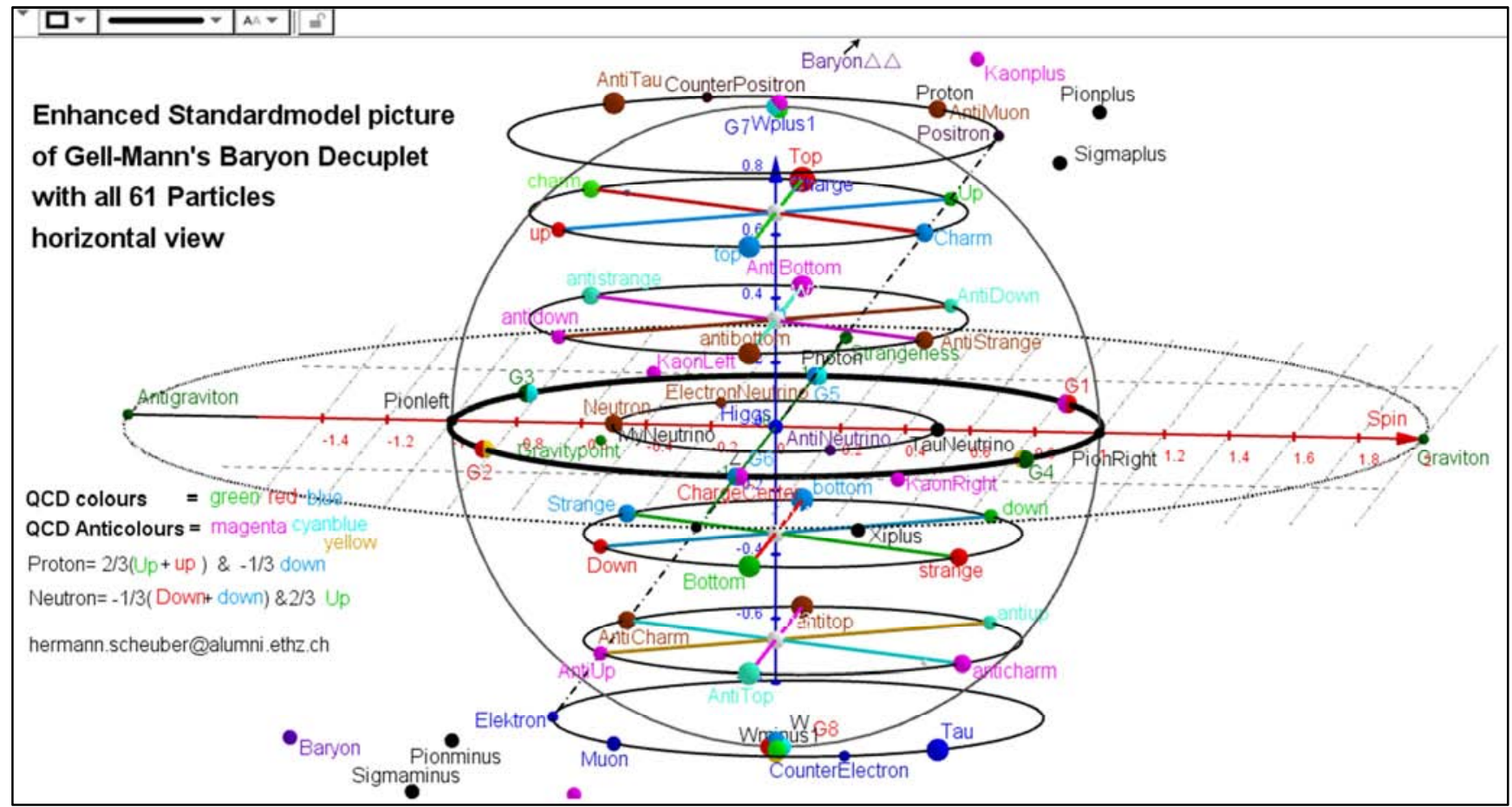

Figure 7. Great Model of the 61 ELEMENTARY PARTICLES with QCD sequent cross particles. 


\subsection{Details of the Standardmodel Constructions}

In the CERN brick box there are only the 17 Fundamental Particles. The Leptons can be symmetrically enhanced through the Higgsparticle and the Quarks through the Chargepoints $2 / 3,1 / 3,-1 / 3,-2 / 3$.

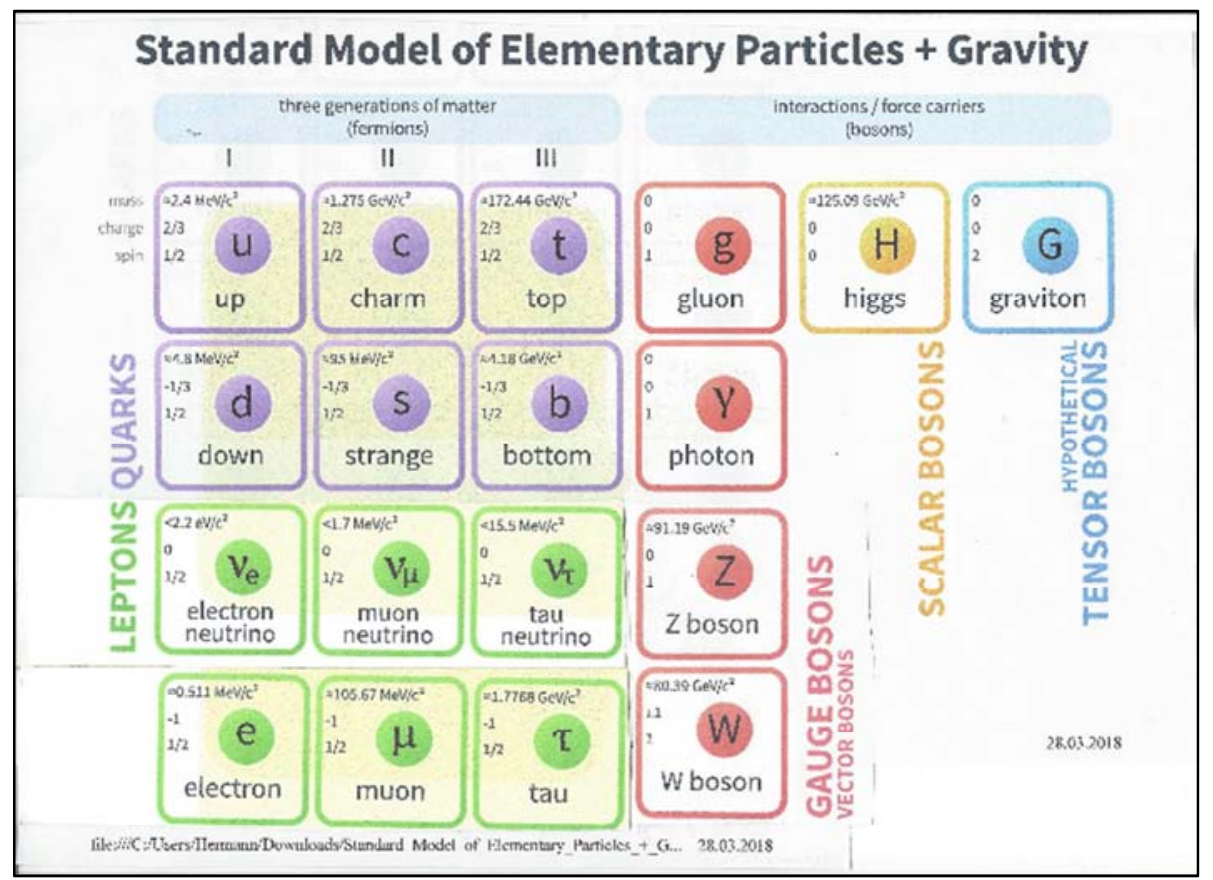

Figure 8. Public Box of the Standardmodel.

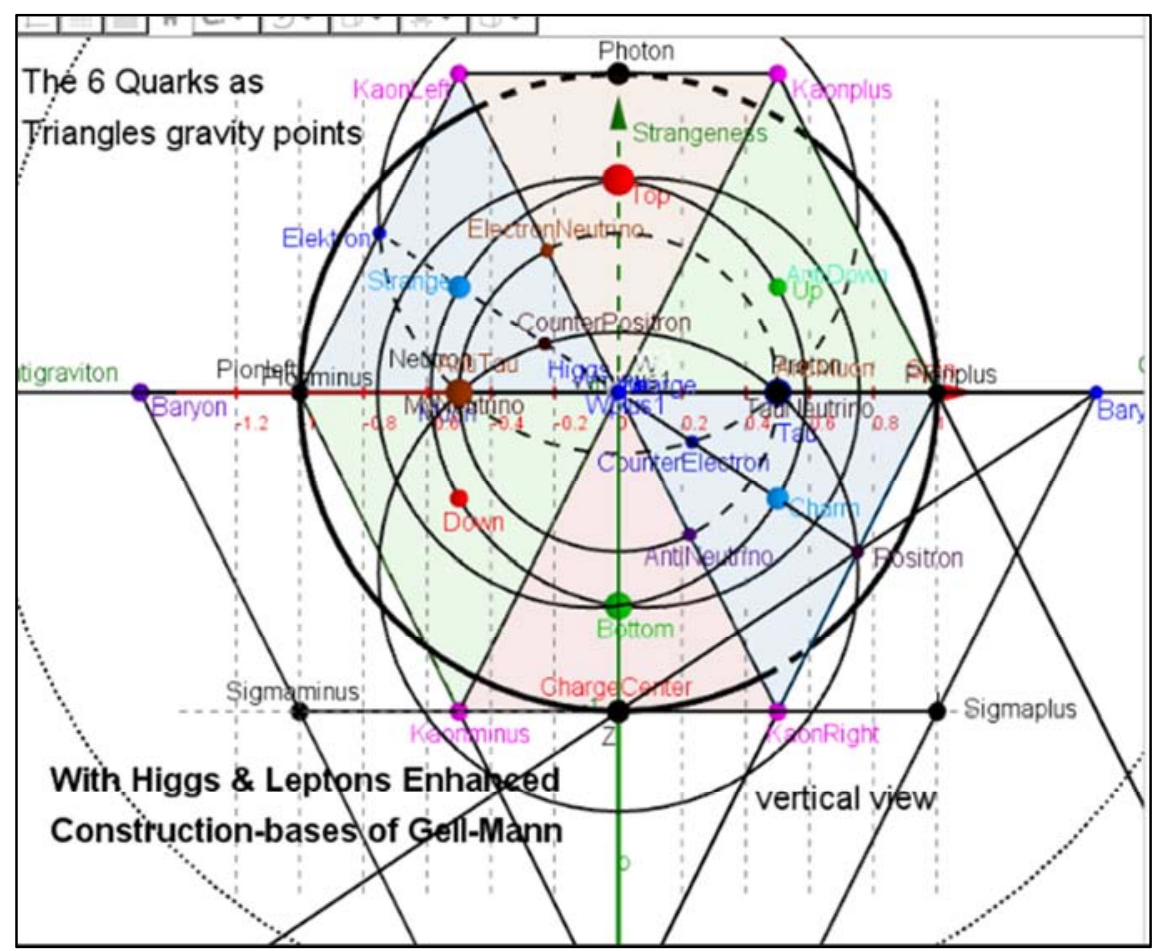

Figure 9. The 6 Quarks as Points of Gravity.

This picture shows that if the Quarks are real (and even the 6 and 2 Gluons) then it is also real that only three particle families can exist! So, a great problem of particle physics should be solved. (In the proved CERN Standard model there is no place for a forth family!)

The 6 Quarks have a real Function: the Gravity points (1/3/2/3 of the Triangle Higgs High-Lines); therefore the 2/3 Charge for the upper $\&-1 / 3$ for the lower Triangles. 


\begin{tabular}{|c|c|c|c|}
\hline 3D Grafik & Konstruktionspro & otokoll & \\
\hline 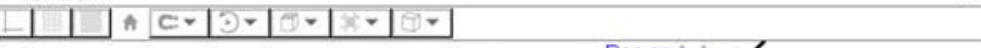 & 田- & (2) & \\
\hline The 12 CFRN I entons & Nr. Name & Beschreibung & Wert \\
\hline Protor/Antimuon Pigeplus Kaonplus & 52 Kreis $d_{2}$ & Kreis durch Antigr... & $\begin{array}{l}d_{2}: X=(0,0,0)+(1.414 \cos (t)+ \\
1.414 \sin (t)-1.414 \cos (t)+141\end{array}$ \\
\hline & 53 Strecke $s$, & Strecke Antigravit.. & $s_{1}=4$ \\
\hline With Higas \& Leptons Enhanced & 54 Punkt Up & & $U p=(0.5,0.333,0.667)$ \\
\hline Construction-bases of Gell-N & 55 Punkt Down & & Down $=(-0.5,-0.333,-0.333)$ \\
\hline Antigrantert- & 56 Punkt Charm & & Charm $=(0.5,-0.333,0.667)$ \\
\hline & 57 Punkt charm1 & & charm1 $=(-0.5,0.333,0.667)$ \\
\hline FI: & 58 Punkt Strange & & Strange $=(-0.5,0.333,-0.333)$ \\
\hline & 59 Punkt Strange 1 & & Strange1 $=(0.5,-0.333,-0.333)$ \\
\hline & 60 Punkt Bottom & & Bottom $=(0,-0.667,-0.333)$ \\
\hline & 61 Punkt Top & & Top $=(0,0.667,0.667)$ \\
\hline & 62 Kreis k & Kreis durch Charm... & $k: X=(-0.084,0,0.667)+(-0.67$ \\
\hline Counter & 63 Kreis q & Kreis durch Strang... & $\mathrm{q}: \mathrm{X}=(0.084,0,-0.333)+(0.672$ \\
\hline uber@alumnı.ett & & $4102 / 10$ & $02 \infty$ \\
\hline
\end{tabular}

Figure 10. Stereo Standardmodel of the CERN brick box.

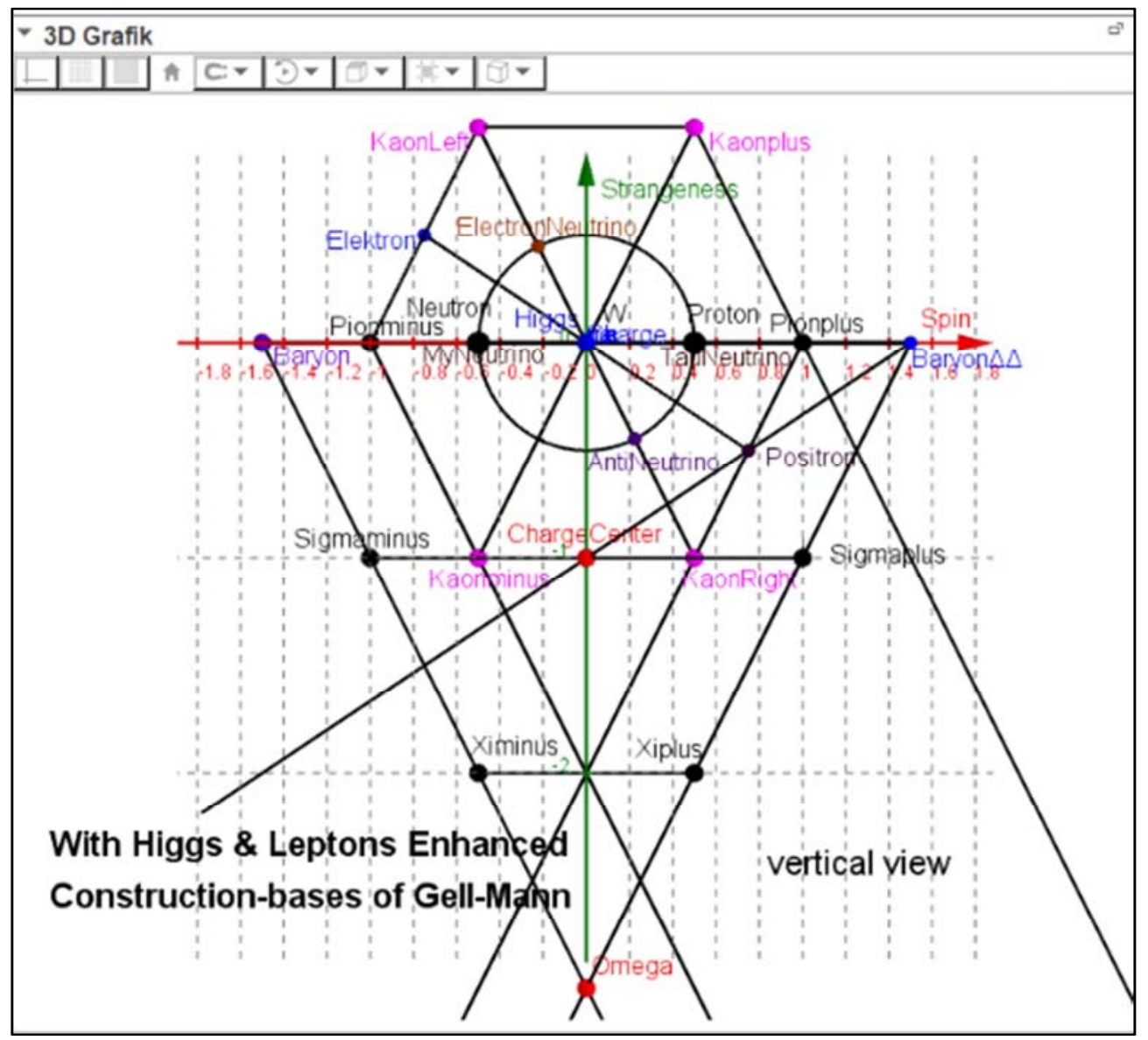

Figure 11. Strangeness $+1,+1 / 2,0,-1 / 2,-1,-2,-3$ in the combined \& enhanced Gell-Mann's Construction.

\section{Discussion}

All these pictures are parts of the Lagrange formula of the enhanced Standard model with the 61 elementary particles, especially for peoples without enough mathematical knowledge getting a deeper insight into the particle physics. 
It is an experiment to demonstrate that the Standard model can be depicted using the Gell-Mann system of matrices to show the mesons (strangeness zero) and the baryon Decuplet with the oblique charge incident.

To get a real tree-dimensional stereo picture, the charge (composed of the $+\&-W$ ray) must be put perpendicular against the horizontal spin. This will be right turning on the right side and left turning on the left side. Connected with the - strangeness on the front side, the 4 tri-pointed charge planes of the CERN Standard model can be put on circles. As they are all half spin particles, according to Pauli they have 3 electron-inverted counter particles on the same plane; and because of the baryons, 3 distinguishable particles are depicted by 3 imaginary colors in the (white) plane center. According to the (Higgs) center point symmetry, the 2 Quark planes must have 2 Antiquark planes, and the Electron plane an Antielectron $=$ Positron plane. The result will then be

7 half spin planes. But as the zero plane cannot be doubled, this special case is shown by the fact that also the Tau-neutrino (on the right spin side) is left turning. This demands a further investigation of the Neutrinos to complete the Standard Model. The upper pictures show that the later discovered particles (Quarks, Gluons \& Higgs) can be fitted into the same model if both $\mathrm{W}+\& \mathrm{~W}$ - rays are united with the Higgs particle in the middle, getting a charge axis. The Quantum Chromo Dynamic is achieved by putting a segment colored e.g. blue between the other both colored green and red spin inverted Quarks, crossing the middle point of that Quark plane. Without the connecting segment a white ball can be put into the center, containing all three colors; but then the missing third Quark color may be written. Only Baryons containing all three Quarks with different colors are stable. For a better overview and for purposes of verification, models without construction lines and helping points can be used, whereby all Quark circle lines must have three times 2 Quark particles with the same color and the white ball in the middle of the Quark plane. It should also be mentioned that heavier particles with greater mass have a greater diameter in the model. The Nomenclature of all the particles may not always be correct, but as each particle has its fixed place, the particle concerned can be found in each language.

As Gell-Mann constructed at the bottom point of his picture with spin 2 the later discovered particle OMEGA, I enhanced the Neutral plane also with spin 2 and put there the very weak force particle, the GRAVITON and antigraviton.

\section{Conclusion}

In conclusion, I propose that the graphical system could provide a better model as an aid to memory as well as for future research rather than a strange mathematical equation with 20 Parameter-constants [5] which is ponderous and difficult to verify investigate. I am wondering why particle physics never published any picture after the investigation of the Higgs particle, but only showed a CERN box of independent bricks and put all the power to speculate and calculate the future SUSY supersymmetry with hypothetical grey matter particles - if the immense mass of the 4 Neutrinos and perhaps even possible 4 vanished anti-matter Neutrinos is not enough. The present construction model of the 61 elementary particles shows all systematically in a real Model and not only in a brick box; thus, from each particle we can see at once 2 Values: Spin $1 / 2$ ( or 1 ), Spin1, Spin2 and Charge $(+1,+2 / 3,+1 / 3,0,-1 / 3,-2 / 3,-1)$ without looking in Wikipedia e.g. [(except for the left turning Tau-neutrino); indication that the system is complete?]

Of course, the stationary particle System gives only a systematic review of all 61 physical particles of the Standard Model; In the baryons e.g., there are only 3 (Anti)Quarks in Confinement with 3 Gluons. But the neighborhood of the wanted particles is conspicuous.

\section{Acknowledgements}

The author is grateful to the support from ResearchGate [10] and Wikimedia for the prepublications.

\section{References}

[1] Mathematics science https://ned.ipac.caltech.edu/level5/Cottingham/frames.html

[2] CERN history https://www.youtube.com/watch?v=cfwBelQubYI

[3] brick box https://en.wikipedia.org/wiki/Standard_Model_(mathematical formulation)

[4] Mathematic formula $1 \mathrm{https} / / / \mathrm{www}$. sciencealert.com/this-iswhat-the-standard-model-of-physics-actually-looks-like

[5] Mathematic formula 2 https://www.symmetrymagazine.org/article/the-deconstructedstandard-model-equation

[6] Eightfold Way - Wikipedia https://de.wikipedia.org/wiki/Eightfold_Way Step by step in video https:/www.youtube.com Standard Model picture QCD

[7] Strangeness - Wikipedia https://de.wikipedia.org/wiki/Strangeness

[8] Meson - Wikipedia https://de.wikipedia.org/wiki/Meson

[9] https://www.researchgate.net/publication/305851350_

[10] Standardmodel with 61 elementary particles in picture

[11] GeoGebra Link vertical construction: https://www.geogebra.org/m/zzbvbect

[12] GeoGebra Link horizontal construction: https://www.geogebra.org $/ \mathrm{m} / \mathrm{n} 3$ beje $4 \mathrm{~m}$ 